\title{
Study on empathetic-pain perception in brain induced by three levels of empathetic-pain perception stimuli
}

\author{
Siti Norhayati Md Yassin*, Nugraha Priya Utama, Maheza Irna Mohamad Salim \\ Clinical Science Department, Faculty of Biosciences and Medical Engineering, Universiti Teknologi Malaysia, Skudai 81310, Johor, Malaysia \\ * Corresponding author: sitinorhayati.my@gmail.com
}

\section{Article history}

Received 30 September 2017

Accepted 7 December 2017

\section{Graphical abstract}

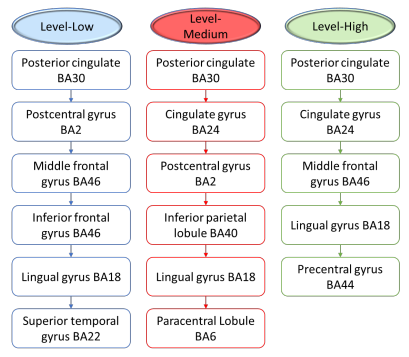

\begin{abstract}
Empathetic-pain perception is a divergence from empathy which is a pain perceived as a reflection of perception from others. The study of empathetic-pain perception and empathy were always related with psychological disorder effecting social and humanity values. The process involved in empathetic-pain perception formations in brain were believed to be different if induced by different level of empathetic-pain perception stimuli. Therefore, this paper was aimed to study the processes involved in empathetic-pain perception formation by revealing the activation-time intervals and source location of the highest empatheticpain perception intensity. This study conducted an experiment to induce empathetic-pain perception on 16 participants using still pictures as visual-stimuli. Electroencephalograph (EEG) recorded brain signal of the participants during the visual-stimuli presentations while the EEG signal were analysed using MATLAB ${ }^{\circledR}$ toolbox, EEGLAB. Time/frequency decomposition in EEGLAB produces ERSP images which determines the activation-time intervals for empathetic-pain perception and, by performing source localization within the activation-time intervals using SLORETA, the source locations for most active processes in empathetic-pain perception were determined. The processes involved in empathetic-pain perception formation in every level were 'stimuli-learning' and 'memory-reconstructions' by Posterior Cingulate BA 30, pain-regulation by either Postcentral Gyrus BA 2, Cingulate Gyrus BA 24 or both, and visual-stimuli and visual-memory processing by Lingual Gyrus at almost similar time intervals. However, the processes were also performed by various brain areas to either perform attention-sustain process while managed working memory and self-control regulation by Middle Frontal Gyrus BA 46, mirror-neurons activation while processed attention information and emotions by Inferior Parietal Lobule BA 40, multisensory integration by Superior Temporal Gyrus BA 22, or motorneurons activation to control the skeletal system respectively in every level by Paracentral Lobule BA 6 and Precentral Gyrus BA44. In conclusion, the empathetic-pain perception formation process discovery were necessary to differentiate every affectional level of the empathetic-pain perception
\end{abstract}

Keywords: EEG, Empathetic-pain, ERSP, IAPS database, source location

\section{INTRODUCTION}

The word 'empathy' originates from Greek word empathia, which comprises of Greek prefix em-, means inside and pathos, means feeling; etymologically, empathy means 'inside feeling' (Lewis, 1991). While, empathy definition as in Compact Oxford Dictionary \& Thesaurus by Waite and Hawker (2009) is 'the ability to understand and share the feelings of someone else'. On the other hand, the definition of empathy in the Oxford Dictionary of Psychology by Colman (2015) is 'the capacity to understand and enter into another person's feelings and emotions or to experience something from the other person's point of view'. Therefore, the definitions of empathy were consummated as a perception which identifies feelings of someone into own self to experience the same affection with that someone.Empathy perception, the social-emotional response that is induced by the perception of another person's affective state, is a fundamental component of emotional experience, and plays a vital role in social interaction (Eisenberg \& Strayer, 1990; Goldstein \& Michaels, 1985; McDonald \& Mesinger). In other words, empathy allow interactions within a community by perceiving emotion which similar with the other person and react to the situation together with them. On the other hand,
Empathetic-pain perception is a derivative adjective from empathy which means, a perceived pain by feelings. In other words, empathetic pain perception is a process that allows someone to feel the pain that is suffered by other people like it is occurring in their own self. To put in other words, empathetic pain perception perceiving brain input without receiving any bodily sensations to them (Borsook \& Becerra, 2009; Campbell-Yeo, Latimer, \& Johnston, 2008; De Vignemont \& Jacob, 2012; Jackson, Brunet, Meltzoff, \& Decety, 2006; Singer et al., 2004).

Empathy perception deficiencies are factor of many psychological illness and mental problems. Some example for the psychological illness and mental problems due to lack of empathy perception are autism, sociopath and psychopath (Baron-Cohen \& Wheelwright, 2004; Jones, Happé, Gilbert, Burnett, \& Viding, 2010). Empathy perception is very important to human life especially towards infants and children. The reason a person should have empathy perception is because empathy perception prevent a person from become 'animal-like' cold-heartless human being (de Waal, 2008; Lynam, Whiteside, \& Jones, 1999). Other than that, failure of perceiving empathy also means that the person either fails to learn or have problems with their memory and emotional perception 
(Benedek \& Schetky, 1987; Bloise \& Johnson, 2007; Goldstein \& Goedhart, 1973; Nugraha P. Utama, Takemoto, Koike, \& Nakamura, 2008; N. P. Utama, Takemoto, Koike, \& Nakamura, 2009). Empathy perception has been studied before by some psychologists and neuroscientists considering that empathy perception falls into cognitive perception category. However, empathy ability is assumed to be varied depends to individuals and are more subjective when it is empathetic-pain. Pain is basically referring to self-standard which some might have high pain tolerance, while some might have low pain tolerance. Therefore, this study aimed to reveal the neurological process during empathetic-pain perception formation induced by three levels of empathetic-pain perception stimuli. The revelation of neurogical process during empathetic-pain perception by different levels of stimuli could provide another option in recognizing empathetic ability by comparing the brain activity with the findings.

Generally, visual stimuli (as it was an observation) information is firstly received by occipital lobe (Heberlein, Padon, Gillihan, Farah, \& Fellows, 2008). Actually, while observing pain of others, we actually feed visual stimuli into primary visual cortex (Kosslyn, Thompson, Kim, \& Alpert, 1995; Maziyar, Yunus, \& Utama, 2015; Molavi, Yunus, \& Utama, 2016; Nugraha P Utama, Takemoto, Nakamura, \& Koike, 2009). The information from the primary visual cortex is passed through the sensory areas following a higher order of the sensory areas. There were two possible pathways that the information might have taken which each of them will go to either parietal association cortex or to temporal association cortex. The pathways are called the dorsal stream and the ventral stream respectively. These pathways of visual information are illustrated in Figure 1.4 below. The dorsal stream carries spatial information of the inputs like its location and dimensions while the ventral stream carries information based on facts of the inputs like its form, character, face mimics, and behaviour. The dorsal stream extends information to dorsal frontal association cortex to help in operates the motor neurons which contained there and besides help in execute control functions of the brain. In the pathway, the second stream brings the information into the temporal association cortex to be recognized and the temporal association cortex projects the information to the ventral regions of frontal cortex (Barbas, 2007; Haynes \& Rees, 2005; Noesselt et al., 2007; Zeki et al., 1991).

\section{MATERIALS AND METHOD}

\section{Participants}

In this study, there were two independent groups of participants which a group is involved in selection of empathetic-pain perception stimuli while the other group were involved in empathetic-pain perception experiment.

The participant group for selection of empathetic-pain perception stimuli is labelled as Group A and is consisted of 50 students from UTM, aged from 19 to 25 years old. The students' agreement to help screening the empathetic-pain inductive pictures were obtained. The gender distribution is 25 females (F) to 25 males (M) (25F:25M). On the other hand, the participants that involved in empathetic-pain perception experiment are belongs to Group B. Group B consisted of 16 healthy students with age ranged from 19-25 years old (MEAN = 22.31 years, $\mathrm{SD}=2.15$ ) with ratio of eight males to eight female students $(8 \mathrm{M}: 8 \mathrm{~F})$. The Group B participants were also involved in electroencephalogram (EEG) recording during the experiment. Prior to the non-invasive measurement involved, the participants were advised to read an informed consent to learn about experiment procedure and psychological risks that they may face after participated in this experiment. In addition, the experiment possesses an ethical endorsement to carry out non-invasive study on brain signals of healthy humans regarding psychophysics stimulation which approved by Research Management Centre of Universiti Teknologi Malaysia. All the participants from both groups are free from any sort of psychological complexes or mental illnesses.

\section{Selection of empathetic-pain perception stimuli}

In this study, the stimuli are obtained from International Affective Picture System (IAPS) provided by The National Institute of Mental Health (NIMH) for the Study of Emotion and Attention from University of Florida (Lang, Bradley, \& Cuthbert, 2005). Still pictures, when used as inducing stimuli in an experiment are called visual-stimuli. In this study, the pictures were screened through two selection processes to finalize visual stimuli with potential to induce empathetic-pain perception

In the first screening process, members of Neuroscience Team from Faculty of Biosciences and Medical Engineering (FBME) in Universiti Teknologi Malaysia (UTM) have discussions to select potential empathetic-pain perception visual-stimuli pictures as many as possible. At the end of the selection process, 157 still pictures that contained unfortunate events like deaths, injuries, illnesses, crimes, surgeries, and sadness were chosen; the team members believed that by watching these unfortunate events will lead the perception of empathetic-pain in most participants.

In second screening process, the 157 pictures were presented to Group A participants. They were briefed about empathetic-pain perceptions to help them differentiate empathetic-pain perception from other kind of perceptions. When ready, the selection process begin by scoring the presented pictures in the range of 0 to 5 with 0 as nil, 1 for the least and 5 for the most effective empathetic-pain perception inducer pictures. At the end of the screening process, the scores obtained for every picture were averaged so that each picture has a final empathetic-pain perception score. The pictures were then divided into six stimuli groups which are Group 1, Group 2, Group 3, Group 4, Group 5, and Baseline Group. The groups were merged accordingly to become three level of empathetic-pain perception stimuli. The division of the pictures is as shown in Table 1.

Table 1 Assignation of empathetic-pain perception scores for each empathetic-pain perception stimuli-picture group and level of empathetic-pain perception stimuli

\begin{tabular}{ccc}
\hline $\begin{array}{c}\text { Final empathetic- } \\
\text { pain perception } \\
\text { scores }\end{array}$ & $\begin{array}{c}\text { Pictures of } \\
\text { empathetic-pain } \\
\text { perception group / } \\
\text { level }\end{array}$ & $\begin{array}{c}\text { Level of } \\
\text { empathetic-pain } \\
\text { perception } \\
\text { stimuli }\end{array}$ \\
\hline 0 & Baseline & Baseline \\
$0.1-0.4$ & Exempted & - \\
$0.5-1.4$ & 1 & Low (LL) \\
$1.5-2.4$ & 2 & Low (LL) \\
$2.5-3.4$ & 3 & Medium (LM) \\
$3.5-4.4$ & 4 & High (LH) \\
$4.5-5.01$ & 5 & High (LH) \\
\hline
\end{tabular}

Through the assignation as in Table 1, the different levels of empathetic-pain perception stimuli were created; Level High (LH) consisted of the highest level of empathetic-pain perception pictures, Level Medium (LM) consisted of intermediate level of empatheticpain perception pictures while, Level Low (LL) consisted of the lowest level of empathetic-pain perception pictures and, Baseline Level (BL) consisted of pictures contained non-living objects with no potential to induce empathetic-pain perception with propose of inducing neutral-perception instead. The total number of picture per one group is ten pictures; the groups were filled started with pictures that have largest score in the group and stopped when the group is fully occupied. However, the number of visual-stimuli to be presented in the experiment are five pictures from each group. Therefore, the visual-stimuli were selected randomly using MATLAB ${ }^{\circledR}$ randomizer. 


\section{Tools and equipment}

The empathetic-pain perception experiment is about presenting visual-stimuli contained painful events to participants to induce empathetic-pain perception in the participants' brain. The visual stimuli used in the experiment are from IAPS database. IAPS is a database of hundreds of colour photographs depicting wildlife, bodily mutilations, landscapes, children, babies, insects, accidents, and many other scenes designed to elicit a range of emotions along the dimensions of pleasure, arousal, and dominance. The earliest version of the database was developed in 1988 by the psychologist from United State Peter J Lang (born 1930) and collaborators at the University of Florida, and it is widely used in research on emotional arousal (Lang et al., 2005). pictures included in the IAPS include selection of a broad sample of contents across the entire affective space, all pictures are in colours, and there must be only easy-toresolve pictures are selected which the pictures must have clear figure-ground relationships, and communicate affective quality relatively without lagging. Therefore, the laboratory members were first pre-screened potential new materials for ease of resolution and affective impact from the pictures. Finally, materials that meet these criteria are then included in the IAPS.

During the empathetic-pain perception experiment, the visualstimuli are presented and graphically designed using MATLAB add-in extension called Psychtoolbox. Psychtoolbox has function to visualize any desired task presentation from MATLAB coding on laptop screen (Brainard, 1997; Kleiner et al., 2007; Pelli, 1997). Thus, the design of the task for this study is written as MATLAB coding and ran using psychtoolbox. Psychtoolbox read the stimuli pictures by IAPS that loaded into it and projected them to become visual-stimuli as designed (Brainard, 1997; Kleiner et al., 2007; Pelli, 1997).Other than that, Hare's Psychopathy Checklist-Revised (PCL-R) is given to each of the participants to assess their psychopathy tendency which directly tell the participants' level of empathy perception. The maximum score of the checklist is 40 where the different total scores inversely define subjective empathetic-pain perceptions of the participants from psychopathy tendency. The high score determines that there are high tendency of psychopathy and that the level of empathy perception is low (Decety, Chen, Harenski, \& Kiehl, 2013). The same case goes when the score for psychopathy tendency is low. Thus, we selected only participants with low PCL-R value $(0 \leq$ PCL-R $\leq 20)$ for further data collection because it means their empathy perception level is high and because participants with low PCL-R score are presumed to have capability in perceive empathetic-pain. The division of the scores for level of empathy perception are as follows: 1) high level of psychopathy tendency or low level of empathy perception $(31 \leq$ PCL$\mathrm{R} \leq 40)$, 2) intermediate level of psychopathy tendency or intermediate level of empathy perception $(21 \leq$ PCL-R $\leq 30)$, and 3 ) low level of psychopathy tendency or high level of empathy perception $(0 \leq$ PCL-R $\leq 20)$ (Hare, Clark, Grann, \& Thornton, 2000).

On the other hand, the brain activities of the participants were recorded using NeuroConn from NEURO PRAX ${ }^{\circledR}$ electroencephalograph (EEG) device. The NeuroConn EEG consisted of Full-band DC-EEG and BIOSIGNAL AMPLIFIER (amplifier) with input impedance larger than $10 \mathrm{G} \Omega$, and a PANEL-PC (therapist). In the experiment, 27 electrode-cables were connected to the 'electrode input box' of the amplifier while the other end of the electrode-cables were attached on NeuroConn EEG cap. The 27 electrodes are positioned on participants' scalp based on extended 1020 system electrode placement. The EEG 10-20 system electrode placement is referring to the distance between adjacent electrodes that were $10 \%$ or $20 \%$ of the distance from anterior to posterior or of the half diameter of the head distance (Chatrian, Lettich, \& Nelson, 1985; Committee, 1994; Jasper, 1958). Other than that, The NeuroConn EEG recorded brain signals at a sampling rate of $4096 \mathrm{~Hz}$ and the recorded EEG data is an electro-physiology signal generated by the brain activity of the participants while watching baseline-set presentation as well as during stimuli-set presentation. During EEG recording, the subject is advised to minimize the unnecessary gestures to reduce artefacts or noise in the EEG data. However, the recorded
EEG data are sensitive to noises and artefacts and required EEG preprocessing to remove the noises and artefacts. Therefore, the EEG data contained empathetic-pain perception activity were trimmed with the same length of every picture and underwent the pre-processing.

Another tool involved in this study is EEGLAB, a toolbox operating in MATLAB environment which offers various functions for continuous and event-related EEG data (Delorme \& Makeig, 2004). Thus, EEGLAB is used to perform the pre-processing procedure of the recorded EEG data. Besides the pre-processing, EEGLAB also involved in the rest of the EEG data analysis in this study using other functions such as re-referencing, artefacts removals, independent component analysis (ICA), and time/frequency analysis (wavelet transform analysis).

Lastly, there is sLORETA, a source localization tool which it applies an instantaneous, distributed, discrete linear solution to extract and depicts information on the time course and source location of EEG brain signal (Pascual-Marqui, 2002).

\section{Design of Empathetic-pain perception experiment}

Empathetic-pain perception could be perceived naturally when we come across an unfortunate event by chance. However, empatheticpain perceptions are induced in this study which is by watching series of empathetic-pain perception visual-stimuli pictures. The experimental design was a modification from experimental design of Utama et.al (Nugraha P. Utama et al., 2008; Nugraha P Utama et al., 2009). The visual-stimuli are presented and graphically designed using Psychtoolbox.

While the participants watching the empathetic-pain perception visual-stimuli in the experiment, data from their brain were recorded. The experiment were designed to have baseline-sets which are from baseline group, and stimuli-sets which are stimuli group in one experiment. There were one baseline-set in front of every six stimulisets which, there were 120 stimuli-sets to be watched by each participant; this make a total of 20 baseline-sets in one experiment. Every baseline-set consisted of a picture of fixation-cross ('+') and five baseline pictures to be watched in a row and same goes to the stimuli-set which also consisted of a picture of fixation-cross and five stimuli pictures per set. The pictures from baseline-sets as well as from stimuli-sets appeared on the screen for $500 \mathrm{~ms}$ and switched to next pictures automatically. Fig. 1 below shows example of picture in baseline group and in every stimuli level.

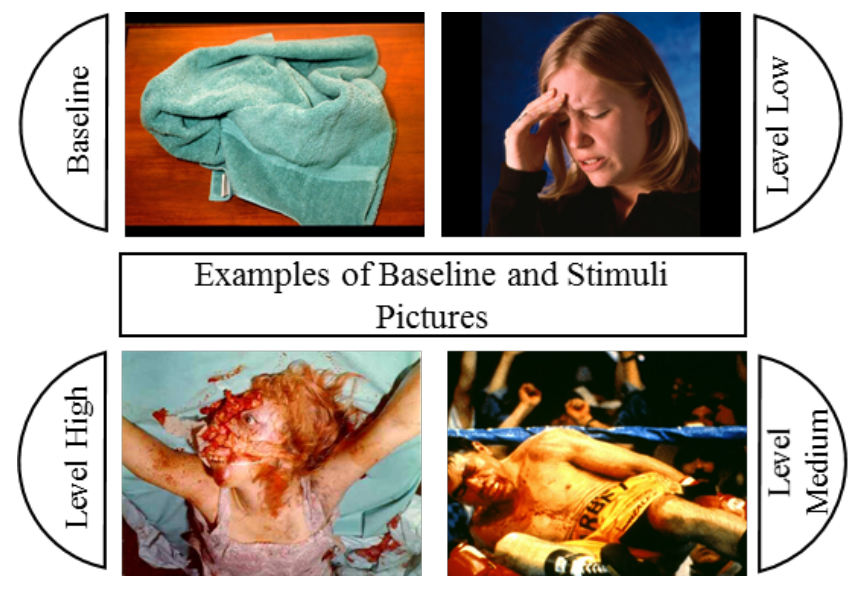

Fig. 1 Examples of still pictures from baseline group and stimuli group in the empathetic-pain perception experiment

\section{Pre-processing of empathetic-pain perception EEG}

During the experiment, the brain activity of participants watching the visual-simuli were recorded at $4096 \mathrm{~Hz}$. In the pre-processing, the EEG data were resampled at $1024 \mathrm{~Hz}$ and were filtered using Finite Impulse Response (FIR) band pass filter of 1-30 Hz; EEG signal is common to have activity within $1-30 \mathrm{~Hz}$. The filtered EEG data were then trimmed to extract data for every individual picture presentation. 
There were $500 \mathrm{~ms}$ of duration for every picture which were averaged for every level separately, as well as for baseline group; every data within each stimuli-level and $\mathrm{BL}$ were averaged to represent respective level or group. The individual data are called 'epoch' or event related EEG data (ERP). Every epoch was loaded with channel location information before performing epoch rejections using extreme values for signals below $-75 \mu \mathrm{V}$ and above $+75 \mu \mathrm{V}$, a typical range for brain activity (Delorme \& Makeig, 2004). Extreme values are bounding values which the data should be within and epochs with data values outsides the extreme values were rejected for examples, facial muscle contraction, and sweating that cause many channels behaving strangely (Escudero, Hornero, Abásolo, \& Fernández, 2011; Woodman, 2010). The epochs left after the rejections were merged for re-reference before performing ICA by EEGLAB as well. The data from each electrode were referenced offline in EEGLAB based on the average signal (Picton et al., 2000). The next procedure following the re-referencing is the ICA. ICA clustered EEG signal and noises separately to create different independent components. The noises were identified by ICA as activities that are frequent, occur at similar times, and are recorded by many channels like eye-blinks noises (Bach \& Jordan, 2002; Hoffmann \& Falkenstein, 2008). Afterwards, the data components were plotted to become 27 components and noisy components as well as components that were seen to have abnormal spectral power were removed and left only cleaned ERP data. Now that the EEG data for that level were cleaned, the EEG pre-processing were complete. The pre-processing steps were performed again with other EEG data of stimuli-set as well as data for baseline-set.

\section{Determination of empathetic-pain perception activation- time}

Wavelet transform analysis is conducted in this study through EEGLAB to determine the activation-time of empathetic-pain perception by plotting time/frequency decomposition of EEG data in EEGLAB. The empathetic-pain perception EEG data (LL, LM, and LH) were averaged separately and were subtracted with neutralperception ERP data (baseline-sets) to let the time/frequency decomposition determine the activation-time of empathetic-pain perceptions over neutral-perceptions. Plotting of time/frequency decomposition produced event-related spectral perturbation (ERSP) image which depicts activation-time based on changes or perturbations in the spectral content of the data. The changes or perturbations were due to empathetic-pain perception activity over neutral-perception activity, which could be positively or negatively triggered.

There are series of colour marks in the ERSP image which represent positive and negative activity of empathetic-pain perception. The colour scale on the right represents positive activity with yellowto-red colour, while negative activity is represented with blueish-toblue colour while zero activity or neutral activity with green colour. Positive activities mean that empathetic-pain perception was higher than neutral-perception while, negative activities of empathetic-pain perception mean that the empathetic-pain perception activities were suppressed to become lesser than neutral-perception activity. The empathetic-pain might be suppressed by other affection that comes along the empathetic-pain perception presentation such as fear, disgust, and anger (Martin et al., 2015). Whereas, neutral activity indicates that empathetic-pain perception has same activity with neutral-perception.

\section{Source localisation of brain activities using sLORETA}

Source localization is performed on ERP files within activationtime intervals which were following the time frames for colour marks in the ERSP images. The ERP data for empathetic-pain perception is transformed into sLORETA format and were statistically analysed in comparison with ERP data for perceptions towards baseline pictures using Log of ratio of averages (log of F-ratio). The statistical analysis produces a generated sLORETA (*.slor) file which contains the test result of significance difference between empathetic-pain perception and neutral perception. The test result files were dragged into
sLORETA Viewer which generating voxel values to represent empathetic-pain perception intensities for voxels that appeared on MRI template. The highest empathetic-pain perception intensities were identified by finding the highest voxel values to investigate the formation of empathetic-pain perception.

\section{RESULTS AND DISCUSSION}

Activation-time of empathetic-pain perception were determined by performing time/frequency decomposition and by tracing the start and end time of coloured-marks appeared on ERSP images. The ERSP images produced for LL, LM, and LH of empathetic-pain perception are shown in Fig. 2, Fig. 3, and Fig. 4 respectively.

\section{ERSP Image for LEVEL LOW (LL) EPP}

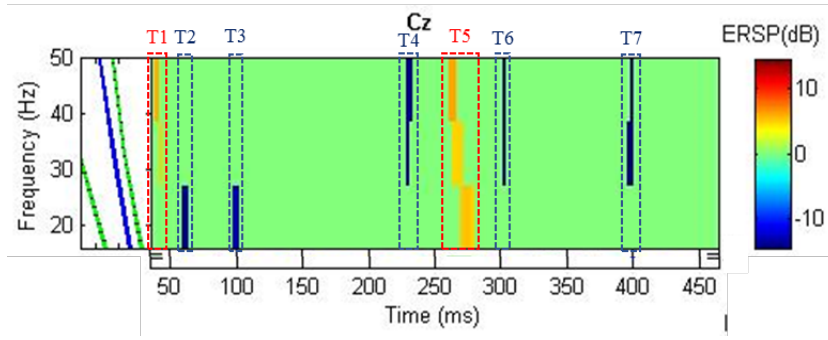

Fig. 2 ERSP image with significant differences between empatheticpain perception and neutral-perception are the activation-time frames for empathetic-pain perception activity of (LL).

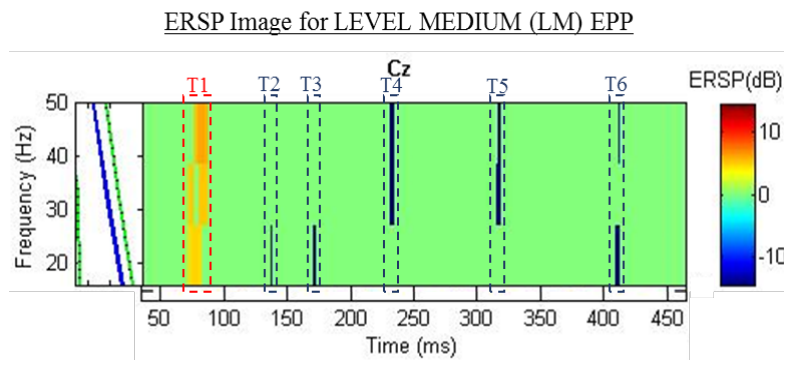

Fig. 3 ERSP image with significant differences between empatheticpain perception and neutral-perception are the activation-time frames for empathetic-pain perception activity of LM.

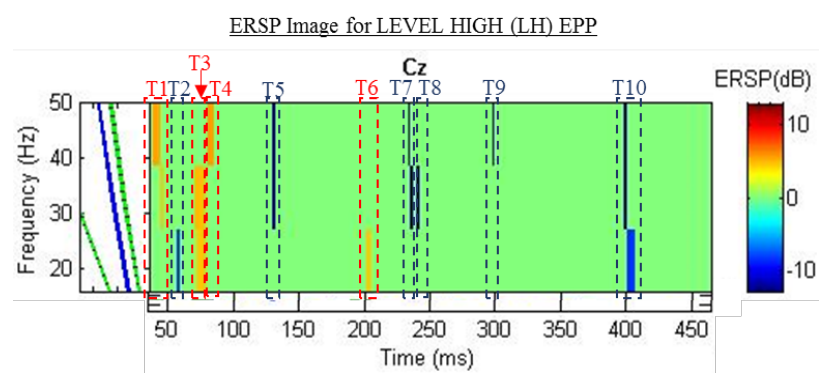

Fig. 4 ERSP image with significant differences between empatheticpain perception and neutral-perception are the activation-time frames for empathetic-pain perception activity of $\mathrm{LH}$

Overall, the ERSP images concluded that perturbations in the spectral content of the subtracted empathetic-pain perception ERP over neutral-perception ERP were recognised as significant difference between empathetic pain perception and neutral-perception activity in brain. In this study, the time interval for the significant difference to take place are activation-time frame for empathetic-pain perception. The empathetic-pain perception activation-time frame were recorded and were essential in comparing empathetic-pain perception process corresponding to different levels of empathetic-pain perception stimuli 


\section{Process of empathetic-pain perception formation in brain}

Following the activation-time intervals of empathetic-pain perception, there were source location of empathetic-pain perception that could describing the formation of empathetic-pain perception in brain to be compared among every level. This study determined most dominant empathetic-pain perception source location by finding brain areas with the highest voxel-value in every activation-time interval of every empathetic-pain perception level from the 'Max/Min' list. Graphs contained time transitions and source location of the most active brain signal while watching the empathetic-pain perception stimuli of every level can be found in Fig. 5, Fig. 6, and Fig. 7 respectively.

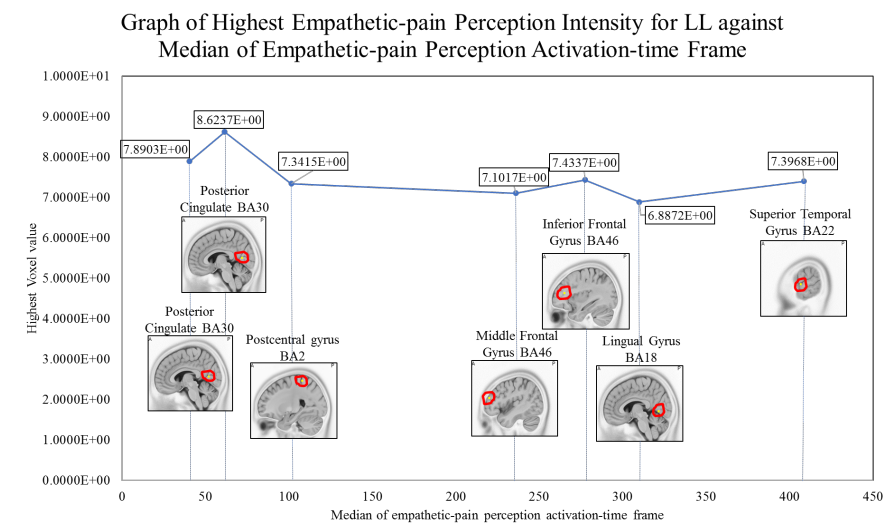

Fig. 5 Graph of empathetic-pain perception formation with the source location of the highest empathetic-pain perception intensity for LL at every activation-time.

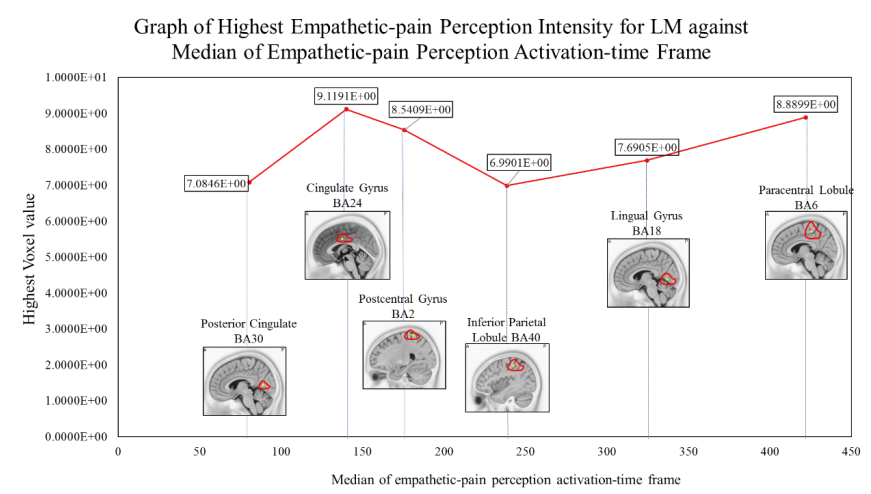

Fig. 6 Graph of empathetic-pain perception formation with the source location of the highest empathetic-pain perception intensity for LM at every activation-time.

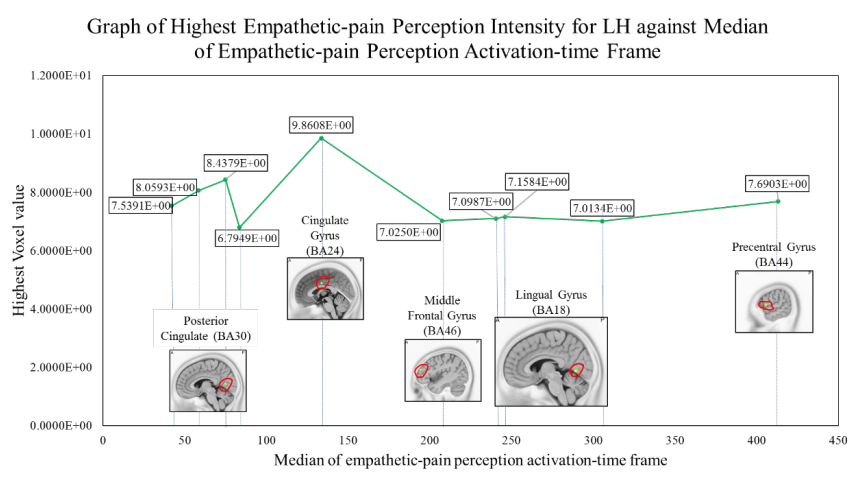

Fig. 7 Graph of empathetic-pain perception formation with the source location of the highest empathetic-pain perception intensity for $\mathrm{LH}$ at every activation-time.
Overall, the most dominated empathetic-pain perception in activation-time interval for every level as seen in Table 4.5 are Posterior Cingulate BA30, Postcentral Gyrus BA2, Middle Frontal Gyrus BA46, Inferior Frontal Gyrus BA46, Lingual Gyrus BA18, Superior Temporal Gyrus BA22, Cingulate Gyrus BA24, Inferior Parietal Lobule BA40, Paracentral Lobule BA6, and Precentral Gyrus BA44. Throughout the $500 \mathrm{~ms}$ time of the experiment, the highest empathetic-pain perception intensities were found in different brain areas among the activation-time intervals.

The discovery of the different brain areas within every activationtime interval throughout the $500 \mathrm{~ms}$ time of the experiment would explain the processes involved in empathetic-pain perception formation for every level (Churchland \& Sejnowski, 2016; Davison, 2014; Dragoi, 2016; Schwaber, 2010). The formation of empatheticpain perception as induced by LL, LM, and LH of empathetic-pain perception stimuli shows that the processes of the highest empatheticpain perception intensity involved are different in every level as every brain area possesses different brain function which can be found in Table 2 follows.

Table 2 Brain function for the brain areas involved in formation of empathetic-pain perception

\begin{tabular}{|c|c|c|}
\hline $\begin{array}{l}\text { Source } \\
\text { location }\end{array}$ & $\begin{array}{l}\text { Associated } \\
\text { stimuli level }\end{array}$ & $\begin{array}{l}\text { Process executed during } \\
\text { empathetic-pain perception }\end{array}$ \\
\hline $\begin{array}{l}\text { Lingual gyrus } \\
\text { BA } 18\end{array}$ & LL, LM, LH & $\begin{array}{l}\text { Processing visual stimuli and visual } \\
\text { memory. }\end{array}$ \\
\hline $\begin{array}{l}\text { Paracentral } \\
\text { lobule BA } 6\end{array}$ & LM & $\begin{array}{c}\text { Premotor cortex; controls motor } \\
\text { activity. }\end{array}$ \\
\hline $\begin{array}{c}\text { Posterior } \\
\text { Cingulate BA } \\
30\end{array}$ & LL, LM, LH & $\begin{array}{l}\text { Involved in emotion, activated by } \\
\text { emotional memory recalling (learning) } \\
\text { and pain. }\end{array}$ \\
\hline $\begin{array}{c}\text { Precentral } \\
\text { gyrus BA 44 }\end{array}$ & LH & $\begin{array}{l}\text { Controls voluntary movements of } \\
\text { skeletal muscles, visual inspection. }\end{array}$ \\
\hline $\begin{array}{l}\text { Postcentral } \\
\text { gyrus BA } 2\end{array}$ & LL, LM & $\begin{array}{l}\text { Sensory receptive area for sense of } \\
\text { touch and pain, a part of } \\
\text { somatosensory cortex. }\end{array}$ \\
\hline
\end{tabular}

Sustaining attention, managing working memory, regulate self-control.

Regulate emotions due to facial stimuli, analyses social information received visually, multisensory integration,

Mirror system, processing information from attention, emotional processing.

Participate in limbic system by
producing anatomic effects associated with emotions

Cingulate

LM, LH

Inferior
Frontal Gyrus
BA 46

Mirror neurons firing, active during motor preparation

Referring to Table 2 above, Postcentral Cingulate BA 30 is a brain area that involved in memory recall and learning. In empathetic-pain perception formation of every level, the empathetic-pain perception pain stimuli were learned and identified within the first $100 \mathrm{~ms}$ by the Postcentral Cingulate BA 30. In previous studies on empathetic-pain perception, Limbic System and Primary Somatosensory Cortex are found to be activated during empathetic-pain perception stimulation and were known as sensory receptive area pertaining to pain which responses emotionally. Among the brain areas listed in Table 2, Postcentral Gyrus BA 2 is one of the Primary Somatosensory Cortex while, Cingulate Gyrus BA 24 is one of the Limbic System. After the regulation by either the Limbic System area or the Primary Somatosensory area, LL and LH of empathetic-pain perception formation performed attention sustaining, working memory 
management, and self-control regulation by Middle Frontal Gyrus BA 46. On the other hand, LM processed information from attention by Inferior Parietal Lobule BA 40 which also hosted the mirror system and emotional processing process. In every level of empathetic-pain perception formation, there is Lingual Gyrus BA 18 as the second-last activated brain area which processed visual stimuli and visual memory. However, the processed information from the Lingual Gyrus BA 18 was transferred to Superior Temporal Gyrus BA 22 for LL, Paracentral Lobule BA 6 for LM, and Precentral Gyrus BA 44 for LH which, all the three brain areas completed the formation using multiple integration of auditory and visual information, motor neurons to control the movements of the skeletal muscles.

Meanwhile, the transfer path of empathetic-pain perception signal for every level in brain were different as well. Empathetic-pain perception signal for LL started at limbic lobe and then, was parietal lobe, frontal lobe, occipital lobe, and temporal lobe. Whereas, empathetic-pain perception signal for LM took a path from limbic lobe, parietal lobe, and ended at frontal lobe. Finally, empathetic-pain perception signal for $\mathrm{LH}$ traveled from limbic lobe to frontal lobe only. The signal transfer path shows that, the higher the empatheticpain perception stimuli level, the shorter the transfer route of empathetic-pain perception signal in brain. The shorter transfer route indicates the short time taken in processing information by the stimuli.

\section{CONCLUSION}

In conclusion, the results in this study have discovered the activation-time, source location, and intensity of empathetic-pain perception activity are not uniform among the levels of empatheticpain perception. The sequence of lobule activated when the empathetic-pain perception signal traveled in brain also difference for every level which made the empathetic-pain perception by LL, LM, and LH stimuli distinct and recognible. Therefore, the proposed hypothesis which suggest that studying the process of empathetic-pain perception formation induced by different empathetic-pain perception are essential for empathy detection, were supported and the objective to provide an option in recognizing empathy ability trough empathetic-pain stimuli was achieved.

\section{ACKNOWLEDGEMENT}

The equipment in this work was provided by Biomedical Materials (B-MEMS) and FBME. Authors would like to acknowledge financial support from UTM RU Grant Q.J130000.2545.15H87.

\section{REFERENCES}

Bach, F. R., Jordan, M. I. (2002). Finding clusters in independent component analysis. Berkeley,California: Computer Science Division, University of California.

Barbas, H. (2007). Flow of Information for emotions through temporal and orbitofrontal pathways. Journal of Anatomy, 211(2), 237-249.

Baron-Cohen, S., Wheelwright, S. (2004). The empathy quotient: an investigation of adults with asperger syndrome or high functioning autism, and normal sex differences. Journal of Autism and Developmental Disorders, 34(2), 163-175.

Benedek, E. P., Schetky, D. H. (1987). Problems in validating allegations of sexual abuse. Part 1: Factors affecting perception and recall of events. Journal of the American Academy of Child \& Adolescent Psychiatry, 26(6), 912-915.

Bloise, S. M., Johnson, M. K. (2007). Memory for emotional and neutral information: Gender and individual differences in emotional sensitivity. Memory, 15(2), 192-204.

Borsook, D., \& Becerra, L. (2009). Emotional pain without sensory paindream on? Neuron, 61(2), 153-155.

Brainard, D. H. (1997). The psychophysics toolbox. Spatial vision, 10, 433436.

Campbell-Yeo, M., Latimer, M., \& Johnston, C. (2008). The empathetic response in nurses who treat pain: concept analysis. Journal of advanced nursing, 61(6), 711-719.

Chatrian, G., Lettich, E., \& Nelson, P. (1985). Ten percent electrode system for topographic studies of spontaneous and evoked EEG activities. American Journal of EEG technology, 25(2), 83-92.
Churchland, P. S., \& Sejnowski, T. J. (2016). The computational brain. MIT press.

Colman, A. M. (2015). A Dictionary of Psychology. USA: Oxford University Press.

Committee, E. P. N. (1994). Guideline thirteen: guidelines for standard electrode position nomenclature. Journal of Clinical Neurophysiology, 11, 111-113.

Davison, M. (2014). Developing an historical empathy pathway with new zealand secondary school students. International Journal of Historical Learning, Teaching and Research, 12(2), 05-21.

De Vignemont, F., Jacob, P. (2012). What is it like to feel another's pain?*. Philosophy of Science, 79(2), 295-316.

De Waal, F. B. M. (2008). Putting the altruism back into altruism: The evolution of empathy. Annual Review of Psychology, 59, 279-300.

Decety, J., Chen, C., Harenski, C., Kiehl, K. A. (2013). An fMRI study of affective perspective taking in individuals with psychopathy: imagining another in pain does not evoke empathy. Frontiers in Human Neuroscience, 7, 489.

Delorme, A., Makeig, S. (2004). EEGLAB: an open source toolbox for analysis of single-trial EEG dynamics including independent component analysis. Journal of neuroscience Methods, 134(1), 9-21.

Dragoi, V. (2016). Visual Processing: Cortical Pathways.: Department of Neurobiology and Anatomy-The University of Texas Medical School at Houston. Retrieved from:

URL: http://nba.uth.tmc.edu/neuroscience/s2/chapter15.html Eisenberg, N., Strayer, J. (1990). Empathy and its development: CUP Archive.

Escudero, J., Hornero, R., Abásolo, D., Fernández, A. (2011). Quantitative evaluation of artifact removal in real magnetoencephalogram signals with blind source separation. Annals of Biomedical Engineering, 39(8), 22742286.

Goldstein, A. P., Goedhart, A. (1973). The use of structured learning for empathy enhancement in paraprofessional psychotherapist training. Journal of Community Psychology, 1(2), 168-173.

Goldstein, A. P., Michaels, G. Y. (1985). Empathy: Development, training, and consequences. Erlbaum Associates, Incorporated, Lawrence.

Hare, R. D., Clark, D., Grann, M., \& Thornton, D. (2000). Psychopathy and the predictive validity of the PCL-R: An international perspective. Behavioral Sciences \& The Law, 18(5), 623-645.

Haynes, J.-D., Rees, G. (2005). Predicting the orientation of invisible stimuli from activity in human primary visual cortex. Nature Neuroscience, $8(5)$, 686-691.

Heberlein, A. S., Padon, A. A., Gillihan, S. J., Farah, M. J., Fellows, L. K. (2008). Ventromedial frontal lobe plays a critical role in facial emotion recognition. Journal of Cognitive Neuroscience, 20(4), 721-733.

Hoffmann, S., \& Falkenstein, M. (2008). The correction of eye blink artefacts in the EEG: a comparison of two prominent methods. PLoS One, 3(8), e3004.

Jackson, P. L., Brunet, E., Meltzoff, A. N., Decety, J. (2006). Empathy examined through the neural mechanisms involved in imagining how I feel versus how you feel pain. Neuropsychologia, 44(5), 752-761.

Jasper, H. H. (1958). The ten twenty electrode system of the international federation. Electroencephalography and Clinical Neurophysiology, 10, 371-375.

Jones, A. P., Happé, F. G., Gilbert, F., Burnett, S., \& Viding, E. (2010). Feeling, caring, knowing: different types of empathy deficit in boys with psychopathic tendencies and autism spectrum disorder. Journal of Child Psychology and Psychiatry, 51(11), 1188-1197.

Kleiner, M., Brainard, D., Pelli, D., Ingling, A., Murray, R., Broussard, C. (2007). What's new in Psychtoolbox-3. Perception, 36(14), 1.

Kosslyn, S. M., Thompson, W. L., Kim, I. J., Alpert, N. M. (1995). Topographical representations of mental images in primary visual cortex. Nature, 378(6556), 496-498.

Lang, P. J., Bradley, M. M., \& Cuthbert, B. N. (2005). International affective picture system (IAPS): Affective ratings of pictures and instruction manual. Retrieved from https://www2.unifesp.br/dpsicobio/adap/instructions.pdf

Lewis, N. (1991). Word power made easy. Simon and Schuster. New York: Pocket Books.

Lynam, D. R., Whiteside, S., Jones, S. (1999). Self-reported psychopathy: A validation study. Journal of Personality Assessment, 73(1), 110-132.

Martin, L. J., Hathaway, G., Isbester, K., Mirali, S., Acland, E. L., Niederstrasser, N., et al. (2015). Reducing social stress elicits emotional contagion of pain in mouse and human strangers. Current Biology, 25(3), 326-332.

Maziyar, M., Yunus, J., Utama, N. P. (2015). The Effect of Ramadan fasting on Visual spatial attention through food stimuli. Research Journal of Medical Sciences, 9(5), 279-288. 
McDonald, N. M., Mesinger, D. S. The development of empathy: How, When, and why Free will, emotions, and moral actions. Philosophy and Neuroscience in Dialogue: In Press.

Molavi, M., Yunus, J., \& Utama, N. P. (2016). The effect of Ramadan fasting on spatial attention through emotional stimuli. Psychology Research and Behavior Management, 9, 105-114.

Noesselt, T., Rieger, J. W., Schoenfeld, M. A., Kanowski, M., Hinrichs, H., Heinze, H.-J., Driver, J. (2007). Audiovisual temporal correspondence modulates human multisensory superior temporal sulcus plus primary sensory cortices. The Journal of Neuroscience, 27(42), 11431-11441.

Pascual-Marqui, R. D. (2002). Standardized low-resolution brain electromagnetic tomography (sLORETA): technical details. Methods and Findings in Experimental and Clinical Pharmacology, 24 Suppl D, 5-12.

Pelli, D. G. (1997). The VideoToolbox software for visual psychophysics: Transforming numbers into movies. Spatial Vision, 10(4), 437-442.

Picton, T., Bentin, S., Berg, P., Donchin, E., Hillyard, S., Johnson, R., et al. (2000). Guidelines for using human event-related potentials to study cognition: recording standards and publication criteria. Psychophysiology, $37(2), 127-152$

Schwaber, E. A. (2010). Reflections on Heinz Kohut's last presentation,"On Empathy," 1981: Its Impact On My Own Pathway. International Journal of Psychoanalytic Self Psychology, 5(2), 160-176.

Singer, T., Seymour, B., O'Doherty, J., Kaube, H., Dolan, R. J., Frith, C. D. (2004). Empathy for pain involves the affective but not sensory components of pain. Science, 303(5661), 1157-1162.

Utama, N. P., Takemoto, A., Koike, Y., Nakamura, K. (2008). Serial processing of emotional type and intensity: Evidence from an ERP Study. In M. Ishikawa, K. Doya, H. Miyamoto \& T. Yamakawa (Eds.), Neural Information Processing: 14th International Conference, ICONIP 2007, Kitakyushu, Japan, November 13-16, 2007, Revised Selected Papers, Part II (pp. 960-968). Berlin, Heidelberg: Springer Berlin Heidelberg.

Utama, N. P., Takemoto, A., Koike, Y., Nakamura, K. (2009). Phased processing of facial emotion: an ERP study. Neuroscience Research, 64(1), 30-40.

Utama, N. P., Takemoto, A., Nakamura, K., Koike, Y. (2009). Single-tria EEG data to classify type and intensity of facial emotion from P100 and N170. Paper presented at the International Joint Conference on Neural Networks (IJCNN 2009). 14-19 June 2009. Atlanta, GA, USA: IEEE, 3156-3163. Waite, M., Hawker, S. (2009). Compact Oxford dictionary and thesaurus. USA: Oxford University Press.

Woodman, G. F. (2010). A brief introduction to the use of event-related potentials in studies of perception and attention. Attention, Perception, \& Psychophysics, 72(8), 2031-2046.

Zeki, S., Watson, J., Lueck, C., Friston, K. J., Kennard, C., Frackowiak, R. (1991). A direct demonstration of functional specialization in human visual cortex. The Journal of Neuroscience, 11(3), 641-649. 